\title{
A Conversation-Analytic Perspective on the Organization of Teacher-Led Clarification and Its Implications for $\mathbf{L} 2$ Teacher Training ${ }^{1}$
}

\author{
Cihat Atar \\ Dr., İstanbul Medeniyet University, Turkey, cihat.atar@medeniyet.edu.tr \\ Paul Seedhouse \\ Prof., Newcastle University,United Kingdom, paul.seedhouse@newcastle.ac.uk
}

1

This study analyses teacher-led clarification sequences in a university second language classroom setting from a conversation-analytic perspective. In the literature, there are many studies of clarification requests, but the focus is on individual categories and quantification. No previous study has examined clarification, as reconceptualised in this study, as a social action using Conversation Analysis methodology. The study draws on 14 hours of L2 classroom data from Newcastle University Corpus of Academic Spoken English database involving international students studying English as a second language. The data is analysed using Conversation Analysis by specifically focusing on sequential organization and the repair mechanism. The findings offer a qualitative and detailed account of clarification from an emic perspective. The analysis suggests that teachers use three types of initiation in clarification and the selection of these types is shown to be strongly related to the extent of epistemic gaps. The analysis also shows that teachers use two further resources when students fail to clarify. These findings have implications for the organization of the repair mechanism in L2 classrooms and for training L2 teachers.

Keywords: clarification, L2 teacher training, conversation analysis, the repair mechanism, language learning

\section{INTRODUCTION}

Second language (L2) classrooms have been a focus of extensive study from the perspectives of different approaches. Initial studies usually focused on grammar and formal aspects, but recently the focus has shifted to communication and interactional

\footnotetext{
${ }^{1}$ This article is written from the Doctorate Thesis 'Atar, C. (2016) The Sequential Organization and Management of Teachers' Other-Initiation of Clarification in Second Language Classroom Contexts' accepted by Newcastle University, the UK.

Citation: Atar, C., \& Seedhouse, P. (2018). A Conversation-Analytic Perspective on the Organization of Teacher-Led Clarification and Its Implications for L2 Teacher Training. International Journal of Instruction, 11(2), 145-166. https://doi.org/10.12973/iji.2018.11211a
} 
skills (Anatolievna et al., 2017). However, the field is still dominated by the approaches that focus on cognition and quantification. As Firth and Wagner (2007) argue, what these approaches lack is a satisfactory focus on interactional and socio-linguistic dimensions of language. A new perspective on L2 classrooms, employing Conversation Analysis (CA) methodology, has initiated a reconceptualization of L2 studies (Kasper \& Wagner, 2011; Sert \& Walsh, 2013). The aim of CA studies of L2 classrooms is to focus on micro details in interaction to understand the actions achieved. This approach enables researchers to analyse data from an emic and inductive perspective which opens up the space for unique observations.

One of the main interactional organizations in CA is the repair mechanism. The repair mechanism is an important tool in understanding how interlocutors deal with troubles in interaction (Liddicoat, 2011). Addressing problems in conversation through repair is an indispensable part of social interaction and thus it is an essential skill for L2 learners. In L2 classrooms students learn how to communicate, but problems do occur frequently and accordingly, it is important to understand how the repair mechanism works so that learners may be scaffolded to learn how to deal with problems (Kasper \& Wagner, 2011). This means that if clarification (CLA), as a repair mechanism, is used in a systematic way, this can give way to more space for interaction and learning (Walsh, 2011).

\section{The Literature on Clarification and Other-Initiated Repairs}

CLA, as used in this study, can be defined as a social action which is other-initiated by teachers to clarify a trouble in a student turn that breaks the mutual understanding between teachers and students (It can also be used for pedagogic purposes according to the institutional goals). Although there are quite a few studies on other-initiated repairs (OIRs) both in first and second language (L2) contexts (Balaman, 2015; Cho \& Larke, 2010; Dingemanse \& Enfield, 2015; Kasper, 2006; Lilja, 2014; Nyroos et al., 2017; Rylander, 2009), CLA is only studied as a by-product of co-construction and it is seen as a strategy. Other-initiated repairs are repair sequences that are initiated by the other interlocutor so that the person who causes an interactional trouble can solve it to achieve intersubjectivity. There are also some studies on clarification requests in Discourse Analysis studies (Ahangari \& Amirzadeh, 2011; Ogino, 2012; Rassaei \& Moinzadeh, 2011). These studies are overly statistical and they do not study CLA in a sequence. Sequential analysis would help researchers in understanding the qualitative aspects and micro details in interaction rather than focusing on making generalizations (Seedhouse, 2004). Finally, there are some CA studies on repair mechanism in L2 contexts (Åhlund \& Aronsson, 2015; Cho \& Larke, 2010; Koshik, 2003, 2005; Rylander, 2009), but problematic moments in L2 classroom interaction is under-researched and none of them studies CLA exclusively. Consequently, it is concluded that there is a gap in the literature in repair studies in L2 classrooms and this study will contribute to the literature not only on L2 classroom interaction, but also on L2 teacher training.

To review some of this literature in more detail, clarification requests from a discourseanalytic perspective may be discussed (Ahangari \& Amirzadeh, 2011; Ogino, 2012; Rassaei \& Moinzadeh, 2011). For instance, Ahangari and Amirzadeh (2011) study 
clarification requests under teachers' corrective feedback. Their aim in their study is to find out which corrective feedback is more useful considering proficiency level differences. Their findings suggest that recasts are the most frequent feedback type in all levels while clarification requests are used more in higher proficiency levels. Their study is a quantitative one and it considers the frequency of each feedback type as the data to rely upon. Thus, this study is overly descriptive and it does not have much account of the interactional features of clarification requests. This means that Ahangari and Amirzadeh's study may be considered as valid if their focus is to look at the frequency of feedback types and compare them. However, a conversation-analytic approach would at least enrich the understanding of how feedback is organized and managed depending on micro details in interaction.

Another study of this type is Ogino (2012). In this study, the objective is to see the effect of modified output and CRs on the language development of learners. The responses of learners for some pre-designed tasks are analysed, but this analysis is again statistical and depends on the frequency of usage. The results suggest that modified output combined with CRs have a moderate positive effect on learners' language development. Consequently, this study concludes that CRs are effective tools and it is a good strategy for teachers to use them to increase language development. However, the interactional features of CRs are not taken into consideration and only the effect of CRs on learners' responses is analysed

Another group that is worth more discussion is the OIR studies in L1 and L2 contexts (Balaman, 2015; Cho \& Larke, 2010; Dingemanse \& Enfield, 2015; Lilja, 2014; Rylander, 2009). Yasui (2010) has specifically studied the phases in repair. Her context is L2 interaction outside classrooms. In her study, she found that there are three phases of repairs. The first phase is the trouble source or the repairable, the next one is the repair-initiation and the final one is the repair or the outcome. Typically, when a problem occurs in talk, this is identified by the speakers and a repair-initiation follows. The next step is performing the repair which is a solution to the problem. Another similar study is Çokal-Karadaş (2010) which focuses on L2 learners' repair use. Her focus is on the assumption that the types of repairs learners use and their proficiency level are related. She does not focus on individual repair types as it is not the focus. However, she makes some claims such as self-initiated self-repairs of students mean more proficiency.

To sum up this part, in these studies CLA is only studied as a by-product of coconstruction and they are seen as a strategy. Some micro features such as the completion of these instances are studied, but the sequential organization of them is not studied specifically and there is no mentioning of CLA as an action, either. Then, it can be concluded from literature review that there is a need to study how CLA is used in L2 classrooms, and how it is organized in terms of sequence organization and the repair mechanism which will contribute to the literature on not only L2 classroom contexts, but also on teacher training. Consequently, it may be argued here that there is a gap in the literature on repair studies in L2 classrooms and the action of CLA, as defined here, is a unique social action described as a result of the emic analysis of the data. To my 
knowledge, no other study has focused on this before. Consequently, this study sets out to unearth the sequential organization and the management of the action of CLA in L2 classroom contexts.

\section{The Repair Mechanism and Intersubjectivity}

In this study, CLA is defined as a repair sequence used to clarify troubles and equalize epistemic gaps in intersubjectivity. Repair in conversation can be defined as 'efforts to deal with trouble sources (TSs) or repairables marked off as distinct within the ongoing talk' (Schegloff, 2007, 101) and intersubjectivity means the mutual understanding betweenlamong participants in interaction. TSs in interaction are anything that break intersubjectivity and prevent interaction from progressing. TSs stem from 3 sources: hearing, understanding and acceptability problems (Schegloff, 2007; Svennevig, 2008).

As mentioned in the definition, CLA is initiated when there is a problem in intersubjectivity. Troubles cause an imbalance in interaction and this imbalance motivates initiating a repair. This means that repair sequences restore shared understanding and this makes the repair mechanism a significant tool in interaction. Heritage $(2012,32)$ argues that an imbalance in interaction means that there is an unknowing " $\mathrm{K}-$-" and a more knowledgeable " $\mathrm{K}+$ " interlocutor and this gap is closed through an epistemic shift as shown below (Heritage \& Clayman $(2010,25))$ :

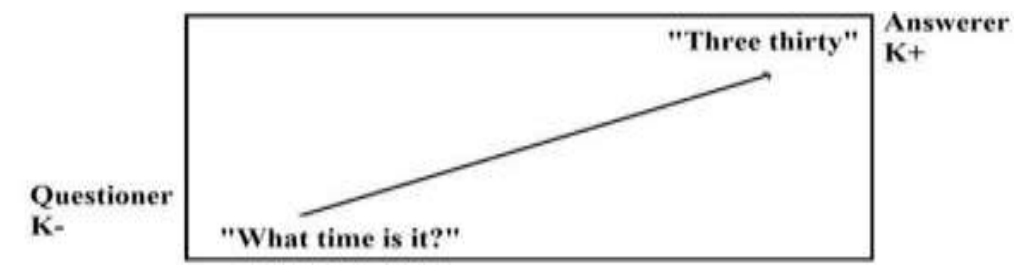

Figure 1

The epistemic shift

Interactional Competence (IC) in L2 classrooms is being adaptive and sensitive to the locally emergent needs in interaction. Therefore, IC can be defined as interlocutors' appropriate use of linguistic and interactional resources to achieve intersubjectivity (Young, 2008). There is much discussion on the nature of IC (Sun, 2014; Young, 2008), but this study follows Markee's IC (Walsh, 2012). The reason is that he focuses on L2 interaction and he has operationalized the concept of IC successfully via the 3 components he has suggested: the formal system (pronunciation, vocabulary and grammar), the semiotic system (turn-taking, sequence organization and the repair mechanism) and gaze and paralinguistic features.

\section{Types of Initiations in Repair}

In the literature, some question types are observed to be used by interlocutors to initiate repair (Kendrick, 2015). Relevant types are open class repair initiators (OCRIs), typespecific questions and partial repetitions followed by a question word $(\mathrm{PR}+\mathrm{WH})$. Other studies (Koshik, 2005) also confirm these types. OCRIs are a repair type which consists of forms such as "huh?", "what?" and "sorry?" and they are described as a weak form of 
repair-initiation (Manrique \& Enfield, 2015) in that they do not identify either the location of the trouble or the source. Another type is type-specific questions (Schegloff, 2007, 78). Unlike OCRIs, they specify the type of information needed. The final type is PR+WHs. This type is an even stronger form of repair-initiator. The reason is a part of the TS is repeated until the problematic part and it is replaced with a question word which strongly locates the trouble.

\section{Resources Used to Deal with Unsuccessful Repair Attempts}

Some TSs cannot be solved by the first initiation and further resources are used to achieve mutual understanding. 2 relevant resources will be reviewed. The first resource is using stronger forms. Multiple repair-initiations are not at random. When the initial repair-initiation fails, the next one tends to be a stronger type. In this way, the trouble is located more precisely. This helps the producer of the TS to find out the problematic part. Checking candidate understanding or hearing is also used after repair failures. It is used to check the current intersubjectivity level to deal with problems (Schegloff, 2000). Shortly, interactants constantly display their mutual understanding and they initiate repair when there is a TS. When the initial repair-initiation cannot clarify the TS, further resources are used to achieve intersubjectivity.

\section{METHOD}

\section{The Purpose of the Study and the Research Questions}

The aim of this study is to investigate teacher-led CLA in L2 classroom settings from the perspective of CA. The originality of this study is based on the research gap in the current literature and the methodology of this study itself. The lack of repair studies on CLA in L2 classrooms and using CA as a methodology to analyse CLA in L2 classroom contexts make this study unique. As discussed in Introduction, it is concluded that CLA as an action was not studied as an integral part of the adjacent sequences, but it was only analysed through a quantitative methodology. In this sense, this study will be the first one to address CLA in L2 classrooms by considering its qualitative features and management. Accordingly, the research questions of the study are as follows:

(1) What are the types of initiations teachers use to initiate CLA?

(2) How is CLA managed when students fail in doing the CLA?

(3) Can the findings of this study be used to train L2 teachers?

Then, this study unearths (1) the initiation types in CLA, (2) the resources teachers use to manage students' failures and (3) the potential of these findings for improving teaching and learning in L2 classrooms.

\section{The Research Context}

The data was collected from L2 classrooms in the INTO department of Newcastle University between 2011 and 2015. The main aim of the INTO department is to help students improve their language abilities to proceed "into" their undergraduate or graduate degree by offering international foundation, international diploma, graduate diploma, English for university study and pre-sessional English. (INTO Courses, 2015). 


\section{The NUCASE Database and the Participants}

Newcastle University Corpus of Academic Spoken English (NUCASE) is a one-millionword spoken academic English corpus. It was recorded in different faculties of Newcastle University and in the INTO English language centre of the university. The data is based on audio and/or video recordings of spoken interaction recorded in seminars, tutorials, $\mathrm{PhD}$ supervisions and staff-student meetings. The sub-corpus INTO database was collected from L2 classrooms and it makes up the last $25 \%$ of the corpus. The recordings in INTO are from pre and in-sessional English classes. The recordings are collected from B1, B2, C1 and C2 levels.

The participants in this study are international students studying at either Foundation or English for University Study programs. The classrooms are made up of 10-12 students. In CA studies, the data and participants are chosen from natural contexts. This is called the specimen approach which is methodologically in contrast to the factist perspective. The underlying assumption of the factist perspective is that a representative sample must be chosen to represent the whole population (Ten Have, 2007). However, in the specimen perspective, participants or contexts are studied as a reality in their natural context. In this sense, the specimen approach is a representation of the reality which is more appropriate for the purposes of this study as the aim of this study is to understand the action of CLA as it occurs in real L2 classrooms. NUCASE data is suitable for this aim as it includes data from natural classroom contexts.

\section{Data Collection}

The aim of this study is to understand how CLA is achieved in interaction. Consequently, the nature of this study requires a qualitative and in-depth analysis of the L2 classroom data. Therefore, the best way of collecting data for this study is to record some L2 classrooms. As mentioned in the previous section, L2 classroom recordings from INTO part of the NUCASE database were used as the data.

As for the amount of data needed for a sound CA study, Seedhouse (2004) states that 5 to 10 hours of classroom data is considered to be adequate for L2 classroom studies and he claims that this data is enough for making generalizations and drawing conclusions about a specific context. Therefore, 14 hours of data from NUCASE were chosen and to balance the distribution of different L2 classroom sub-contexts, 4 lessons from writing classes and 2 lessons from listening and speaking classes are chosen to create the main data of this study. The reason for this choice is that in order to understand the organization of CLA, it is essential to have data from different L2 classroom contexts.

\section{Conversation Analysis}

In this section CA, as a research methodology, will be presented and why it is an appropriate methodology for this study will be justified. CA is one of the several approaches that study spoken language. CA emerged from sociological studies and it is founded upon the ethnomethodology of Goffman and Garfinkel, who studied members of a society and their practices to understand how they interact as social beings (Ten Have, 2007). Garfinkel tried to figure out how ordinary people achieve their 
interactional goals in everyday life. Similarly, Goffman is the pioneer researcher in studying human interaction in close detail (Gardner, 2004). These two researchers paved the way for Harvey Sacks and Emanuel A. Schegloff in the early 1960s. Thanks to their studies, CA turned into a distinct discipline over time and it evolved into a naturalistic approach whose primary aim is to observe, describe, analyse and understand talk as a basic component of human social behaviour (Sidnell, 2010).

In 1960, three basic underlying principles were borrowed from ethnomethodology. These principles are accountability, indexicality and reflexivity (see Gardner, 2004, 266 for more details). In accordance with these basic principles of ethnomethodology, CA has basically three main aims. These are applying the principles of ethnomethodology to naturally occurring talk, unearthing the organization and order of social actions in interaction and understanding how participants in interaction create and orient to a shared understanding (Ten Have, 2007). These main principles of CA were taken into consideration for the data analysis and they shed light on how the data of this study was analysed. The first aim of CA suggests that natural talk is the appropriate type of data if the aim is to study talk-in-interaction. So, in this study the data comes from genuine L2 classrooms. There is no outside intervention on variables and the classrooms were recorded naturally. Secondly, to uncover the actions that teachers and students do in classrooms, the data is analysed via the emic perspective of CA. The initial observations showed that CLA is ordered and patterned as an action in natural interaction. Consequently, in accordance with CA's aims, this study aims at uncovering the order and organization in the action of CLA.

CA has many advantages as a methodology for analysing L2 classroom interaction and considering the aim of this study, it is clear that it is the most appropriate methodology for this study. The reason is it focuses on actions rather than individual functions offering a better and fuller account of the data. It also provides richer details about the data unlike top-down methodologies and it is evidence-based which means that it only studies what can be shown or proven in the data. It allows the researchers to have an open mind for any findings in the data by rejecting pre-defined categories and assumptions unlike Discourse Analysis. The final significant advantage is that CA studies natural talk as the data and this, in turn, prevents researchers from studying artificial language use. There are also some disadvantages. These issues are about the generalizability issue, the amount of data that CA can analyse and the observer's paradox. The researchers of the study took some measures to deal with these limitations. These measures are including as big an amount of L2 classroom data as possible, and also including different sub-contexts, to have a generalizable and adequate amount of data. Making the recording process as unobtrusive as possible is the precaution to decrease the effect of the observer's paradox. These issues will also be addressed in reliability and validity section in more detail.

\section{Data Analysis}

\section{Transcription}

Transcription is the process of creating the orthographic representation of the recordings. Transcription is a very significant part of CA analysis as it is the initial step 
in converting the data into a format by which micro analysis can be undertaken. In this study, the transcription conventions suggested in Liddicoat (2011), which is used in most CA studies (see Appendix A), is used to have a consistent transcription system. It is essential to have a consistent transcription system in a study as it not only assures a reliable representation of the data, but also it allows readers and other researchers to understand the extracts easily. External information such as the lesson type, pedagogic focus and the task type are also added to the transcripts when necessary.

\section{Data analysis procedures}

In this sub-section, the steps of data analysis in CA will be introduced and how sequence organization and the repair mechanism are used to analyse data will be explained.

Seedhouse (2004, 38-39) suggests the following steps for data analysis in CA to account for the organization of an action:

- Unmotivated look at the data

- An inductive search throughout the database to establish a collection of instances of the phenomenon

- Establishing regularities and patterns in relation to the occurrences of the phenomenon in order to show that these instances are produced and oriented to by the participants as normative organization of the action

- Finally, a more generalized account of how the phenomenon relates to interaction in the broader sense is produced.

In accordance with the steps suggested by Seedhouse (2004), the initial data analysis showed that there is a pattern in the action of CLA and this pattern is ordered. In other words, the organization of the action was an observable one as evidenced by the systematic production and by the orientation of the participants. Hence, an extensive literature review was undertaken on CLA as detailed in the Introduction part. Then, a more detailed data analysis was undertaken to understand the sequence organization and the forms and functions of the action. After that, the action was studied from the perspective of repair. Finally, the instances were studied to understand how CLA failures are managed and the implications for $\mathrm{L} 2$ teacher training were mentioned.

To sum up the steps followed in this study, first, the resources teachers use to initiate CLA were studied via sequential organization of CA. Finding out the types of CLAinitiation answers the first research question. After that, the resources that were used to manage CLA failures were studied which answers the second research question. Finally, the initiations and resources teacher used were studied from an IC (Markee, 2008) perspective in order to see if any implications for teacher training can be obtained.

\section{Validity and Reliability}

The validity and reliability of any data collection and data analysis methods are of great importance for the findings of a study (Dornyei, 2007). Therefore, issues about validity and reliability were taken seriously in this study and measures were taken to diminish negative effects. The validity of a research involves making accurate inferences and interpretations from the data. As a qualitative methodology, CA's validity mostly rests 
on the analysis of researchers. As Seedhouse (2004) suggests, the validity of CA analysis is related to what the researcher claims and to what extent the data supports it. In this sense, the researcher of this study did an extensive reading in the initial stages and he presented the findings in different academic circles. The data was firstly presented in MARG (Micro Analysis Research Group) in Newcastle University. MARG is a data session group meeting where researchers studying CA present their data, and participants and researchers discuss the findings. The data was also presented twice in Newcastle University postgraduate conference and also at BAAL 2015 conference which is the biggest Applied Linguistics conference in the UK. By presenting the data and findings, useful feedback from other researchers were obtained and their confirmation of the findings also increased the validity of the study.

The other term, reliability, can be defined as the consistency of the analysis and results of the data. As mentioned in the Research Methodology section, the fact that CA can analyse only a limited amount of data may be a limitation for reliability. The reason is, it may not make strong generalizations or claims depending on a little amount of data. However, CA is strongly interested in details and it makes assumptions about only the things that can be evidenced from the data. Therefore, CA findings consist of what is in the data rather than deductive assumptions or theories. This is directly related to CA's being an empirical and evidence-based approach. In this sense, CA has a valid point of analysis and it does not try to make claims beyond what is in the data (Seedhouse, 2004). The criticism for this limitation of CA is also undermined by recent CA studies. The growing number of findings in CA studies shows that casual talk and institutional talk have observable organization and the basic mechanism in conversation is very similar in different related contexts. In other words, conventions and conversational rules may show some differences in different contexts, but the basic rules of sequence organization or turn taking procedures are usually the same. For instance, in Seedhouse's (1998) study, sub-contexts in L2 classrooms sometimes have different organization, but the basic machinery is the same. Consequently, CA has the ability to make generalizations. In this study, the data comes from L2 classrooms and it is suggested that the interaction in L2 classrooms are organized (Seedhouse, 2004). In this sense, the findings of this study are generalizable to other similar contexts. In addition, Seedhouse (2004) suggests that by sharing the transcripts of the data with readers, the study becomes replicable as the readers can check the accuracy of the analysis.

Another measure taken by the researchers was to include two cameras and two audio recorders. In this way, recordings from the classrooms provided accurate and richer details. Also, the cameras and audio recorders were set up before the classes started to decrease observer's paradox. In addition, the researcher left the classrooms during the lessons to allow students and teachers to behave as naturally as possible. This increases the reliability and the validity of the data as it yields more natural data. Moreover, different sub-contexts and different language skills were included in the data to have more reliable results. Having different L2 sub-contexts made it possible to check whether the findings are parallel in different sub-contexts. Finally, as Seedhouse $(2004,319)$ suggests, "There is no substitute for detailed and in-depth analysis of individual sequences; interviews with participants, questionnaires etc. are not able to provide this, which is why triangulation is not normally 
undertaken". Namely, CA is an adequate approach for studying interaction on its own. As explained in Research Methodology section, CA has many benefits for studying natural interaction and it is a competent methodology on its own for the purposes of this study. Consequently, considering the focus of this study, triangulation is not undertaken.

\section{FINDINGS}

85 extracts were analysed using a micro-analytic perspective. 6 of these extracts were used in this paper to demonstrate the main observations in teacher-led CLA. The findings will be presented in three section each of which is dedicated to a research question.

\section{Initiating CLA}

In this part, the CLA initiation types will be under examination. In the data, teachers were observed to use 3 types of initiation in CLA which are: type-specific questions, OCRIs and PR+WHs. The observations about these different initiation types will be presented answering the adapted CA question 'why these different initiation types, right now?'. The argument here is that teachers use these different types in accordance with the level of epistemic gap. Extract 1 will demonstrate type specific CLA initiations and Extract 2 will introduce OCRIs. Then, Extract 3 will demonstrate PR+WHs.

Extract 1 demonstrates the use of type specific questions by teachers to initiate CLA. In this extract, there is a whole class brain storming activity on culture shock.

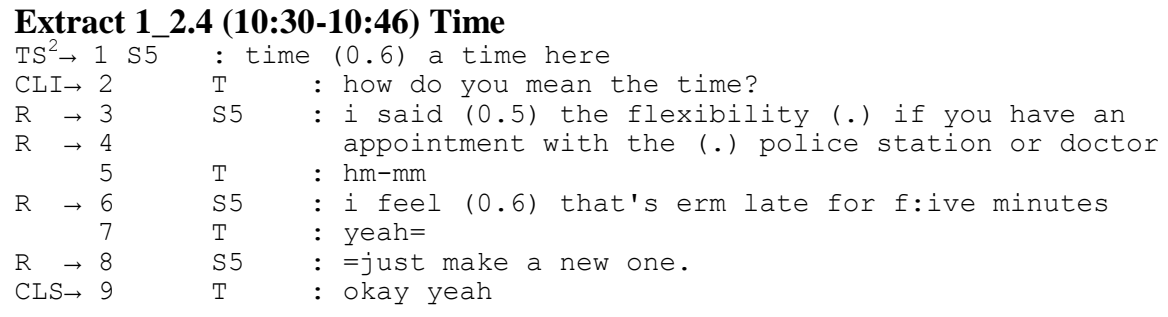

In line $1 \mathrm{~S} 5$ mentions time as a culture shock. The teacher does not clearly understand how time can be a cultural difference and asks S5 to explain it (how do you mean the time?). In the following lines S5 clarifies how time is different for him by mentioning the strictness in the timing of appointments. The teacher closes the turn using sequence closing thirds (okay) and (yeah) (Schegloff, 2007).

In this extract, the teacher considers the student answer in line 1 as a response to his previous initiation. However, he does not know (how) exactly it can be a culture shock. Therefore, he initiates CLA in line 2 with the type-specific question (how do you mean the time?). S5 orients to CLA-initiation by explaining how time is a culture shock for him. In this sense, the teacher uses a certain type of question to let the student know the

${ }^{2}$ Abbreviations in the extracts: CLI: CLA initiation, R: repair|CLA, CLS: closing. 
type of the problem. In response, S5 clarifies his problematic turn and in line 9 the teacher closes the turn which shows that S5's turn in line 1 is now clear.

Type-specific CLA-initiations are repair initiators with questions such as 'how?', 'where?' and 'why?'. They are observed to be the most common type of initiation in the data (slightly more than half of the instances). The observations suggest that typespecific questions typically follow understanding problems as seen in Extract 1 and they are used to clarify problems which 'partially' block intersubjectivity. For instance, in Extract 1 the teacher understands the student's response, but he cannot clearly make out 'how' time is a culture shock. This means that there is some mutual understanding, but there is still a partial problem.

Extract 2 demonstrates OCRIs as CLA initiators. In this extract, the teacher checks any vocabulary that is difficult for the students (here empirically).

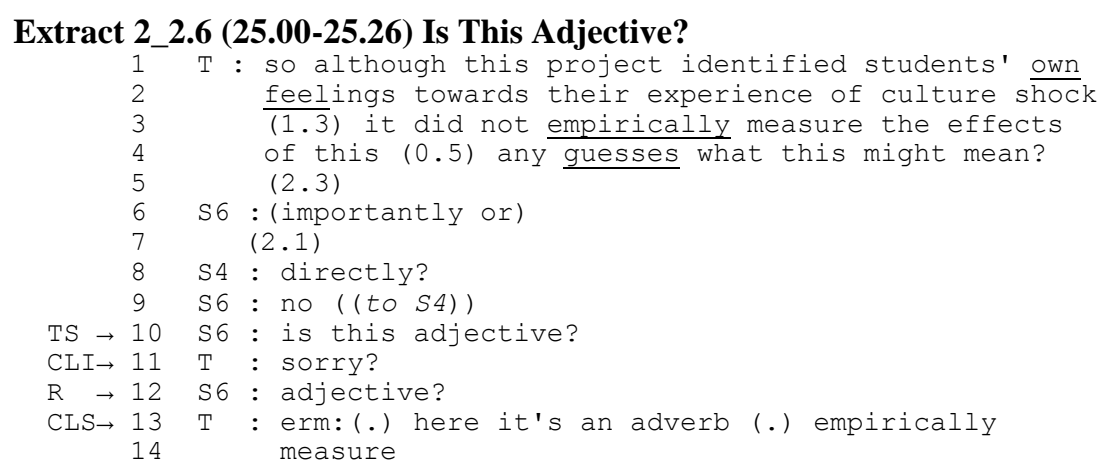

In lines 1 to 4 the teacher reads the relevant part from the text and he allocates the turn to the whole class. In line 6, S6 tries to provide an answer, but there is no explicit acknowledgement or rejection from the teacher. In line 10 rather than providing a guess about the meaning of the word, S6 initiates a new turn to get some additional information about the word. However, the teacher has a difficulty in hearing his response and he initiates CLA using an OCRI. Following the initiation, S6 clarifies the TS by repeating his problematic turn shortly and the teacher's response follows in line 13.

OCRIs are the second most common CLA initiator type in the data (one-fifth of the instances). The most common type of OCRIs is 'sorry' and occasionally 'huh' is observed. OCRIs are used in instances where the teacher has a difficulty in, usually, hearing a previous turn. For instance, in Extract 2 there is a hearing problem and the teacher initiates CLA with an OCRI to show that their mutual understanding is at stake. S4's repair in line 10 shows that he also takes the repair-initiation as an indicator of a hearing problem as he only repeats the TS.

Extract 3 demonstrates the final initiation type, PR+WHs. In this extract, there is a discussion on the functions of the paragraphs in an article. 


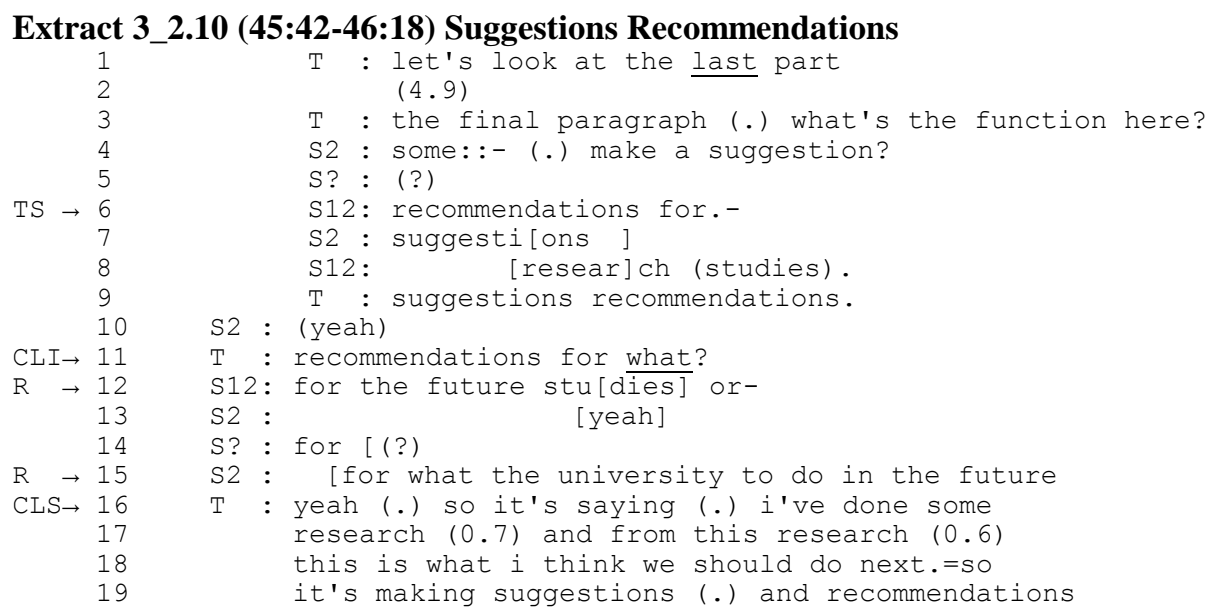

In line 3 the teacher makes an initiation. From line 4 to 8 some students offer answers. In line 9 the teacher repeats the responses however, in line 11 he initiates a repair with (recommendations for what?) to ask students to clarify what the recommendations are for. In lines 12 and 15 S2 and S12 self-nominate and clarify that they are (for the future stu[dies]) and ([for what the university to do in the future). In line 15 the teacher acknowledges the responses.

This extract illustrates how PR+WHs work as CLA initiators. As seen line 9, the teacher acknowledges students' answers as appropriate, but he wants students to clarify what the recommendations are for. This type precisely indicates where the problem is and it is used in both hearing and understanding problems unlike the previous two types. Consequently, the CLA responses tend to be short turns, usually consisting of a few words or a phrase as seen above. Also, as seen in lines 12 and 15, both student CLAs start with 'for', which is a type-conforming response (Schegloff, 2007). This clearly shows that students provide not only the necessary CLA, but also an appropriate phrasal response type.

In this section, the findings on the 3 CLA initiation types are presented and it has been argued that these different types target different levels of epistemic gap and that they are related to specific TSs (hearing or/and understanding).

\section{Management of Students' CLA Failures}

Sometimes students cannot achieve CLA after the initial CLA initiation. In response, teachers are observed to use 2 resources to manage these failures: using stronger forms and checking candidate understanding. The analysis shows that students' CLA failures happen in two main ways: The first one is when a student cannot provide a CLA at all. This is when there is no CLA or when the provided CLA is totally irrelevant. The second one is when students cannot provide a satisfactory CLA. The researchers use the term 'CLA failure' for both observations as the analysis suggests that there are not significant differences between the two in terms of organization. 
Extract 4 demonstrates the use of stronger forms to deal with students' CLA failures. In this extract, students have finished a fill in the blanks activity and the teacher checks if they have any problems with the activity.

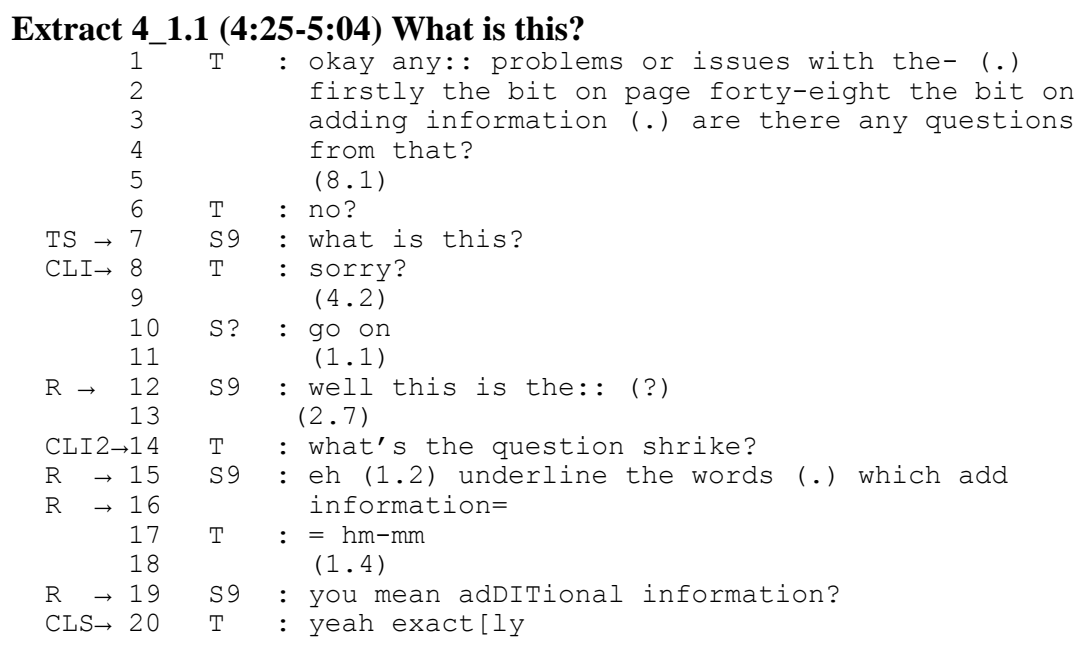

In lines 1 to 4 the teacher asks the students if they have any problems with the activity. There is a long silence of 8.1 seconds and the teacher takes the turn again and asks if there are no questions. S9 asks (what is this?) to show the problem he has encountered. However, the teacher has difficulty in understanding and he initiates CLA with an OCRI. There is a 4.2 seconds pause following the CLA initiation. After a 1.1 second silence, S9 tries to do the CLA, but he cannot complete his sentence. Another pause, 2.7 seconds, follows and in line 14 the teacher re-initiates CLA using a type-specific question (what) this time. In line $15 \mathrm{~S} 9$ starts to do the CLA and in line 19 he continues and clarifies the TS. The teacher confirms the clarified answer and the sequence closes.

This extract illustrates how teachers manage CLA failures using stronger forms. The TS in this extract stems from S9's question in line 7. S9 uses deictic reference 'this' in his turn, but the teacher's following CLA initiations show that it is not clear for the teacher. Hence, to clarify what S9 means, the teacher initiates CLA using an OCRI first. The following 4.2 seconds is a significant silence and it shows that there is a problem. The analysis in this study suggests that silence has a significant role in the interpretation of CLA failures. Students' significantly long silence is often observed in CLA failures and they are interpreted as a CLA failure by teachers. Consequently, as S9 cannot produce CLA and there is a significantly long silence, the teacher interprets CLA failure and reinitiates with a stronger one: a type-specific question. It locates the problem more precisely and this makes the problem clearer for S9. Considering the fact that S9 can do the CLA after the re-initiation, it can be argued that the teacher's strategy has worked.

Checking candidate understanding is the latter resource. The analysis suggests that it used after only partial CLA failures. It is not observed when there is no CLA as there should be some message first to check candidate understanding. Extract 5 provides an 
instance where the teacher checks candidate understanding to see if the CLA done by the student is the same as his/her understanding. In this extract, the interaction is not related to a task, it started simultaneously due to S3's misunderstanding.

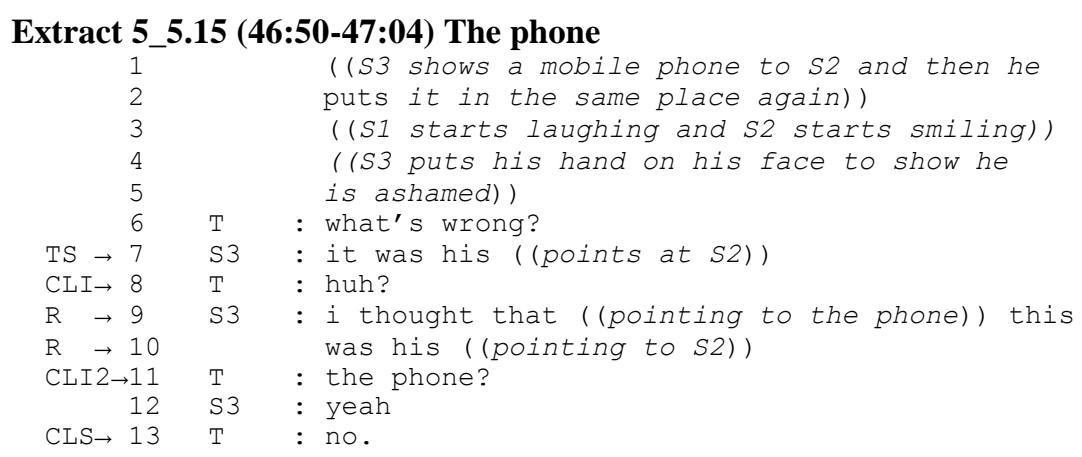

$\mathrm{S} 3$ shows a mobile phone to $\mathrm{S} 2$ and he puts it in the same place. He wants to ask S2 if the mobile phone is his. Then, in line $3 \mathrm{~S} 1$ starts laughing loudly and S2 also smiles. In line 4 S3 covers his face with his hand suggesting that there is an awkward situation. In line 6 realizing that there is something going on, the teacher asks (what's wrong?). In line $7 \mathrm{~S} 3$ tries to explain the situation, but the teacher has a trouble and initiates CLA with (huh?). S3 does the CLA pointing at both S2 and the mobile phone. However, with the confirmation check (the phone?), the teacher still tries to clarify if he is referring to the mobile phone and this is confirmed by S3.

This extract illustrates how checking candidate understanding works as a resource. Via this resource, the teacher makes it sure that they share the same understanding after S3's CLA in lines 9 and 10. If there were a problem, S3 would reject the teacher's candidate understanding and do another CLA (see Extract 6). Finally, the teacher responds with (no.) to S3's response in line 7 to mean that the mobile phone does not belong to S2 which suggests that now the teacher has understood the initial problematic turn.

Now, an example of checking candidate understanding where the candidate understanding is wrong and rejected will be demonstrated. In Extract 6 the topic is culture shock and students first do small group discussion. Then, they have a whole class brain storming activity.

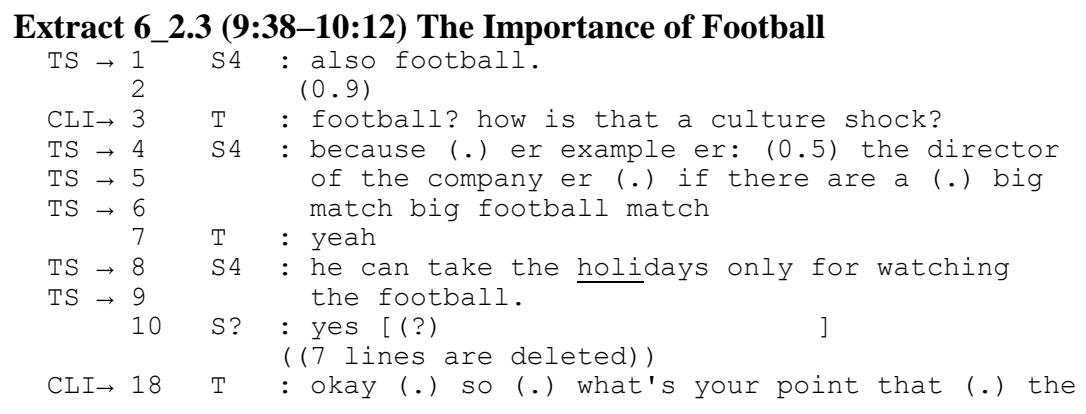




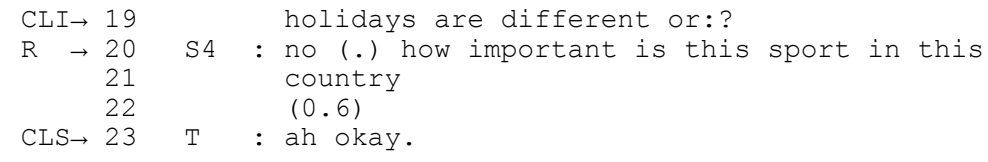

S4 mentions football as a culture shock. In line 3 the teacher claims understanding by repeating the answer, but then, he initiates CLA to have S4 explain how this is a culture shock. From line 4 to $9 \mathrm{~S} 4$ clarifies how football is a culture shock for him. However, the teacher's checking candidate understanding in line 18 shows that what is different is not clear. In line $20 \mathrm{~S} 4$ rejects the candidate understanding and directly goes on to do the CLA by explaining that it is the importance of sports in that country (the UK).

This extract clearly illustrates how checking understanding works as a resource to deal with problems in doing a repair. When there is no problem, it works as a confirmation check (Extract 5), but if the other speaker recognizes a problem, it is understood as a repair initiation as demonstrated in line 20 where $\mathbf{S} 4$ directly starts to do the CLA just after rejecting the teacher's candidate understanding. This shows that S4 takes it as a repair initiator. After S4's CLA, the teacher uses the change-of-state marker 'ah' which is a strong indicator of epistemic change and moving into a $\mathrm{K}+$ (knowing) position (Heritage, 2012). To conclude, checking candidate understanding is like a switch in interaction: if there is no problem in intersubjectivity, it is confirmed by the other interlocutor. However, if a TS is detected, it directly acts as a repair initiator.

\section{DISCUSSION}

This study has reconceptualised CLA as an action through an emic and unmotivated look at the data in accordance with the gap in the literature presented in the Introduction. In accordance with the research questions, 3 points have been analysed using the microanalytic tool of CA: (1) The three types of initiations in CLA, (2) How CLA is managed when there is a CLA failure and (3) the implications for teacher training. The analysis of these points suggests that: (1) Teachers use 3 types of initiations in CLA, type-specific questions, OCRIs and PR+WHs, and these types are related to the level of epistemic gaps. (2) Teachers use 2 further resources to manage students' CLA failures: using stronger forms and checking candidate understanding. The third research question will be discussed with regard to the findings in research questions 1 and 2 at the end of this section.

The first research question unearths the types of initiations in CLA. The analysis shows that teachers use 3 initiation types which are type-specific questions, OCRIs and PR+WHs. The analysis has demonstrated that the extent of the epistemic gap is a significant determiner in the use of different types (Extracts 1, 2 and 3). These initiators are organized in the epistemic engine of Heritage and Clayman (2010) as follows: 


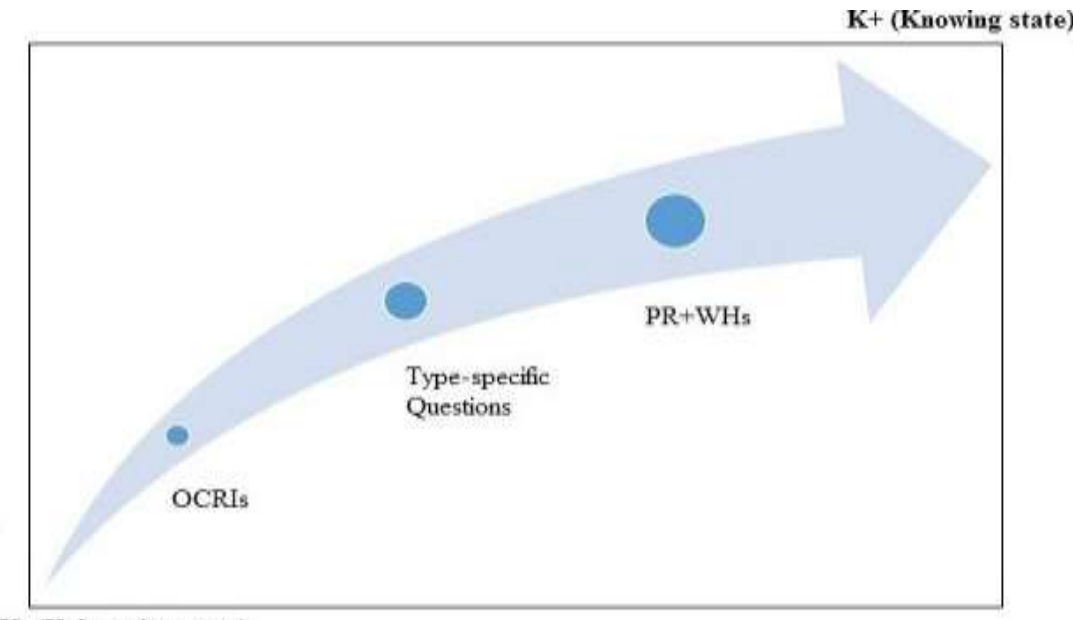

K- (Unknowing state)

Figure 2

Types of initiation with regard to the level of epistemic gap

The analysis of the different initiation types in accordance with the adaptation of the CA question 'why these different initiation types, right now?' suggests that the different types of initiations, as illustrated in Figure 2, are directly related to the extent of epistemic gaps. The Analysis section has demonstrated how teachers use different types to neutralize different levels of epistemic gaps. For instance, in Extract 1 there is a partial problem (closer to $\mathrm{K}+$ ) and the teacher uses a type specific question. However, he uses an OCRI as he literally has no idea about what the student says in Extract 2 (closer to K-).

In response to the second research question, the analysis has demonstrated that teachers use 2 resources (stronger forms and checking candidates) to achieve intersubjectivity. In this study teachers use stronger forms to locate the TS better and the very high ratio of success in CLA failures after the use of stronger forms is evidence that they help achieve intersubjectivity. The findings about candidates in this study have some unique properties, though. In the literature, checking candidates are reported to target both hearing and understanding problems: In fact, they are mentioned separately as checking candidate understanding and checking candidate hearing. However, this study has found that candidates are almost always used for only understanding problems (Extract 5 and 6). Overlaps are a common cause for hearing problems and consequently, it may be argued that this may be the case as there are fewer overlaps in this context. The lessons in the data are more like traditional classrooms where the Initiation-Response-Feedback pattern is observed frequently as the pedagogic goal in the lessons is to improve academic English. Therefore, the participation structures in these classrooms are quite limited and the interaction is often between two people (a teacher and a student) and it is often one way (teachers initiate and students respond). Accordingly, this might lead to fewer overlaps which decrease hearing problems. 
To sum up, the use of 3 different initiation types is an IC and it is the first component (formal system) of Markee's IC (Walsh, 2012). Similarly, the 2 resources teachers use to manage CLA failures show that teachers actively evaluate the current level of epistemic gap and use further resources in case of failures which is a part of the semiotic system of IC. Finally, as discussed in candidates, checking candidate understanding is also a very strategic move which is a part of teachers' IC. It ensures that there is clear mutual understanding and it helps achieve intersubjectivity when there is a problem.

\section{IC and Teacher training}

As the previous section mentioned teachers' IC, displays of teacher ICs and their implications for L2 teacher training will be mentioned here. As analysed and discussed above, teachers use 2 resources to manage CLA failures: stronger forms and checking candidate understanding. In line with previous studies, teachers in this study use stronger forms to deal with troubles. As shown in Extracts 4, teachers recycle the CLAinitiation in a stronger form to locate a problem more precisely. This shows that teachers actively evaluate the current level of epistemic gap and use stronger forms to achieve intersubjectivity much more effectively which is a part of the second component (the semiotic system) of Markee's (2008) IC. It may also be considered as contributing to learning as it helps students achieve CLA and make long explanations. Consequently, the students are scaffolded to produce longer turns and they express themselves better which in turn provides an environment for more interaction and L2 learning. Candidate understanding is also a very strategic move which is a part of teachers' IC. It ensures that a clear mutual understanding between a teacher and a student, and it helps achieve intersubjectivity when there is a problem in mutual understanding.

Now, two implications for teacher training will be discussed here: reflective practice and the management of interaction in L2 classrooms.

Reflective practice has gained popularity especially in recent decades. The logic behind reflective practice is that teachers can improve the quality of their teaching by doing reflective practice (Karim et al., 2017; Ratminingsih et al., 2017; Walsh, 2011). Walsh's SETT (Self Evaluation of Teacher Talk) framework is developed specifically for this purpose and it aims at increasing teachers' language awareness in a systematic way. Recently, some researchers such as Seedhouse $(2008)$ and Walsh $(2006,2011)$ have pointed out the benefits of using CA and micro analysis to do reflective practice. CA can show how interactional style affects students' responses. Hence, teachers can improve their teaching through reflective practice which creates better interaction in L2 classrooms and this facilitates L2 learning (Walsh, 2011).

Considering the value of reflective practice, the analysis of CLA in this study may provide some implications for L2 teachers. First, in Data Analysis it has been shown that initiating repair (here CLA) is not at random and the resources used in CLA sequences are patterned. As shown in the Extracts, the resources used by teachers are sensitive to the TS and the level of the epistemic gap. Therefore, teachers may improve their questioning and repair-initiation skills by analysing and reflecting upon their own use. If they study instances where CLA does not move smoothly or where students have 
difficulty in doing the repair, teachers may check if using another resource would be a better move. For instance, using OCRIs may cause problems as they are vague for locating the epistemic gap and also, they do not mention if there is a hearing or understanding problem. Using a type-specific question, for example, may be a better resource especially in understanding problems. Consequently, the findings of this study may be used in reflective practice to increase teachers' awareness of their use of appropriate repair initiation types. However, it must be kept in mind that, as argued in this thesis, the achievement of intersubjectivity is a local one and it is constructed in a moment by moment fashion.

Also, CA is used to improve L2 teaching and learning by studying L2 classroom interaction (Walsh, 2011). The findings regarding the resources used by teachers for CLA failures can be used to improve the management of interaction in L2 classrooms. For instance, using stronger forms is shown to be an effective resource (Extract 4) and the analysis demonstrates that students are usually able to do the CLA when the teacher re-initiates CLA-initiation using a stronger form. Therefore, it may be suggested that their use as a resource seems to work as seen from the next successful CLA moves of students.

Finally, the resource checking candidate understanding is observed to be quite commonly used by teachers in this study for managing failures. Using candidate understandings may be useful in L2 classrooms as teachers can keep track of intersubjectivity by candidate understandings which enables them to check mutual understanding occasionally. When there is a problem, candidates work as a CLA initiator and students do the CLA automatically (Extract 6). In this sense, teachers may use them to pre-empt possible problems in interaction which may cause further problems and break progressivity later in interaction. Consequently, this can be used to improve teacher trainees' teaching. For instance, as a part of reflective practice, teacher trainers can record teacher trainees' lessons and they can work on these lessons to improve questioning skills through the findings on CLA as explained above. Teacher trainers can show the trainees how different types of initiations work and how some of them can be better than others in certain contexts.

To sum up, the findings of this study regarding CLA initiators and the resources used in CLA failures have some implications for L2 classrooms: increasing teachers' awareness regarding the possible options that have different uses in interaction. The different types of initiation and the resources used for management of failures can be used to increase teachers' awareness and they can be aware of the range of options available for managing problems in interaction. Consequently, by doing reflective practice and using CA to understand the micro mechanisms in interaction, teachers may improve their IC which in turn has the potential to improve their interactional skills. This has the potential to facilitate students' learning and improve their interactional skills. It may also be added that the contribution of this study to reflective practice studies is that the findings of this study draws attention to the first component of Markee (2008) (the formal system) unlike the seminal studies such as Walsh (2011) which usually focuses on the semiotic system. 


\section{CONCLUSION}

The focus of this study, CLA, is studied from a CA perspective in contrast to the quantitative studies in the discourse-analytic tradition. In this sense, this study addresses to the call for studies focusing on more qualitative and social aspects of L2 classroom interaction. The findings have shown that there are 3 types of CLA initiation which are related to the level of epistemic gaps. The findings also demonstrate that 2 further resources are used by teachers in case of student CLA failures. So, this study contributes to L2 repair mechanism studies and improving teachingllearning in L2 classrooms.

As for the limitations of the study, the observations about non-verbal phenomena were not presented due to word limitation. Also, this study focused on only teacher-led CLA and students' initiation of CLA is not studied. Finally, this study aimed at mainly describing and analysing CLA and using the findings to train L2 teachers. Accordingly, studying the development of students' CLA ability could shed light on L2 learning which would be a significant contribution to the field.

\section{REFERENCES}

Ahangari, S., \& Amirzadeh, S. (2011). Exploring the Teachers use of spoken corrective feedback in Teaching Iranian EFL Learners at Different Levels of Proficiency. Procedia - Social and Behavioral Sciences, 29, 1859-1868.

Åhlund, A., \& Aronsson, K. (2015). Corrections as Multiparty Accomplishment in L2 Classroom Conversations. Linguistics and Education, 30, 66-80.

Anatolievna, S. T., Munirovna, N. A., Kasimovna, V. D., Mirzayanovna, S. L., \& Anatolievn, B. T. (2017). Pedagogical Management of University Students' Communication Ability Development. International Journal of Instruction, 10(3), 7992.

Atar, C. (2016). The Sequential Organization and Management of Teachers' Otherinitition of Clarification in Second Language Classroom Contexts (Doctor of Philosophy). University of Newcastle upon Tyne, Newcastle, the United Kingdom.

Balaman, U. (2015). Collaborative construction of online L2 task accomplishment through epistemic progression. Procedia - Social and Behavioral Sciences, 199, 604612.

Cho, E. H., \& Larke, P. J. (2010). Repair Strategies Usage of Primary Elementary ESL Students: Implications for ESL Teachers. The Electronic Journal for English as a Second Language, 14(3), 1-18.

Çokal-Karadaş, D. (2010). Conversational Repair in Foreign Language Classrooms: A Case Study in a Turkish Context. Eurasian Journal of Educational Research (EJER), 39, 145-160.

Dingemanse, M., \& Enfield, N. J. (2015). Other-initiated repair across languages: towards a typology of conversational structures. Open Linguistics, 1, 96-118. 
Dornyei, Z. (2007). Research Methods in Applied Linguistics. Oxford: OUP.

Firth, A., \& Wagner, J. (2007). Second/Foreign language learning as a social accomplishment: Elaborations on a 'reconceptualised' SLA. The Modern Language Journal, 91(1), 800-819.

Gardner, R. (2004). Conversation Analysis. In Davies, A. and Elder, C. (ed) (2004) The Handbook of Applied Linguistics, Blackwell Publishing: Oxford, 262-284.

Heritage, J. (2012). The Epistemic Engine: Sequence organization and territories of knowledge. Research on Language and Social Interaction, 45(1), 30-52.

Heritage, J. \& Clayman, S. E. (2010). Talk in Action: Interactions, Identities and Institutions. Boston: Wiley-Blackwell.

INTO Courses (2015). Retrieved 01 July 2015 from http://www.intohigher.com/uk/engb/our-centres/into-newcastle-university/studying/our-courses.aspx

Karim, A., Mohamed, A. R., \& Rahman, M. M. (2017). EIA- A Teacher Education Project in Bangladesh: An Analysis from Diversified Perspectives. International Journal of Instruction, 10(4), 51-66.

Kasper, G. (2006). Beyond repair: conversation analysis as an approach to SLA. AILA Review, 19, 83-99.

Kasper, G., \& Wagner, J. (2011). A conversation-analytic approach to second language acquisition. In D. Atkinson, (ed.) Alternative Approaches to Second Language Acquisition. London: Routledge, 117-142.

Kendrick, K. H. (2015). The intersection of turn-taking and repair: the timing of otherinitiations of repair in conversation. Frontiers in psychology, 6, 250.

Koshik, I. (2003). Wh-questions used as challenges. Discourse Studies, 5(1), 51-77.

Koshik, I. (2005). Alternative questions used in conversational repair. Discourse Studies, 7(2), 193-211.

Liddicoat, A. J. (2011). An Introduction to Conversation Analysis (Second Edition). Continuum International Publishing group.

Lilja, N. (2014). Partial repetitions as other-initiations of repair in second language talk: Re-establishing understanding and doing learning. Journal of Pragmatics, 71, 98-116.

Manrique, E. \& Enfield, N. J. (2015). Suspending the next turn as a form of repair initiation: evidence from Argentine Sign Language. Frontiers in Psychology, 6, 1326.

Markee, N. (2008). Conversation Analysis. Lawrence Erlbaum Associates, NJ 07430.

Nyroos, L., Sandlund, E., \& Sundqvist, P. (2017). Code-switched repair initiation: The case of Swedish eller in L2 English test interaction. Journal of Pragmatics, 120, 1-16. 
Ogino, M. (2012). Modified output, clarification requests and developmental progress of learner language: the case of negation of adjectives in L2 Japanese. New Zealand Studies in Applied Linguistics, 18(1), 7-22.

Rassai, E., \& Moinzadeh, A. (2011). Investigating the Effects of Three Types of Corrective Feedback on the Acquisition of English Wh-question Forms by Iranian EFL Learners. English Language Teaching, 4(2), 97-106.

Ratminingsih, N. M., Artini, L. P., \& Padmadewi, N. N. (2017). Incorporating Self and Peer Assessment in Reflective Teaching Practices. International Journal of Instruction, 10(4), 165-184. https://doi.org/10.12973/iji.2017.10410a.

Rylander, J. (2009). Repair work in a Chinese as a foreign language classroom. In H. Nguyen \& G. Kasper (Ed.), Talk-in-interaction: Multilingual Perspectives, 245-280.

Schegloff, E. A. (1997). Practices and actions: boundary cases of other-initiated repair. Discourse Processes, 23(3), 499-545.

Schegloff, E. A. (2000). When 'others' initiate repair. Applied Linguistics, 21(2), 205243.

Schegloff, E. A. (2007). Sequence Organization in Interaction. Cambridge, UK: CUP.

Seedhouse, P. (1998). CA and the analysis of foreign language interaction: a reply to Wagner. Journal of Pragmatics, 30, 85-102.

Seedhouse, P. (2004). The Interactional Architecture of the Language Classroom: A Conversation Analysis Perspective. Malden, MA: Blackwell.

Seedhouse, P. (2008). Learning to talk the talk: Conversation Analysis as a tool for induction of trainee teachers. In Garton, S. and Richards, K. (Ed.) Professional Encounters in TESOL. Basingstoke: Palgrave Macmillan, 42-57.

Sert, O., \& Walsh, S. (2013). The interactional management of claims of insufficient knowledge in English language classrooms. Language and Education, 27(6), 542-565.

Sidnell, J. (2010). Conversation Analysis: An Introduction. Wiley-Blackwell.

Sun, D. (2014). From Communicative Competence to Interactional Competence: A New Outlook to the Teaching of Spoken English. Journal of Language Teaching and Research, 5(5), 1062-1070.

Svennevig, J. (2008). Trying the easiest solution first in other-initiation repair. Journal of Pragmatics, 40 (2), 333-348.

ten Have, P. (2007). Doing Conversation Analysis (2nd Edition). London: Sage.

Walsh, S. (2006). Investigating Classroom Discourse. New York: Routledge.

Walsh, S. (2011) Exploring Classroom Discourse Language in Action. Oxon: Routledge. 
Walsh, S. (2012). Conceptualising Classroom Interactional Competence. Novitas-Royal, 6(1), 1-14.

Yasui, E. (2010). Repair and language proficiency: Differences of advanced and beginning language learners in an English-Japanese conversation group. Texas Papers in Foreign Language Education, 14(1), 41-57.

Young, R. F. (2008). Language and Interaction: A Resource Book. Oxon: Routledge.

\begin{tabular}{|c|c|}
\hline & TRANSCRIPTION CONVENTIO \\
\hline$[$ tex & : Indicates the start and end points of overlapping talk \\
\hline$=$ & : Indicates that a certain word/s is immediately followed by others \\
\hline & : Indicates falling pitch or intonation \\
\hline ? & : Indicates rising pitch or intonation \\
\hline & : Indicates an abrupt halt or interruption in utterance \\
\hline underline & : Indicates the speaker is emphasizing or stressing the speech \\
\hline & : Indicates prolongation of a sound \\
\hline (text) & : Speech which is unclear or in doubt in the transcript \\
\hline ((italic)) & : Annotation of non-verbal activity or some explanation \\
\hline (.) & : A brief pause, usually less than 0.2 seconds \\
\hline (123) & : A number in parenthesis indicates the time of a pause in seconds \\
\hline$(?)$ & : Unintelligible speech \\
\hline S? & : Unidentified student \\
\hline
\end{tabular}

